\title{
EL SABER PEDAGÓGICO EN LA FORMACIÓN DOCENTE DE LOS PROGRAMAS DE LICENCIATURA DE LA UNIVERSIDAD DE LOS LLANOS*
}

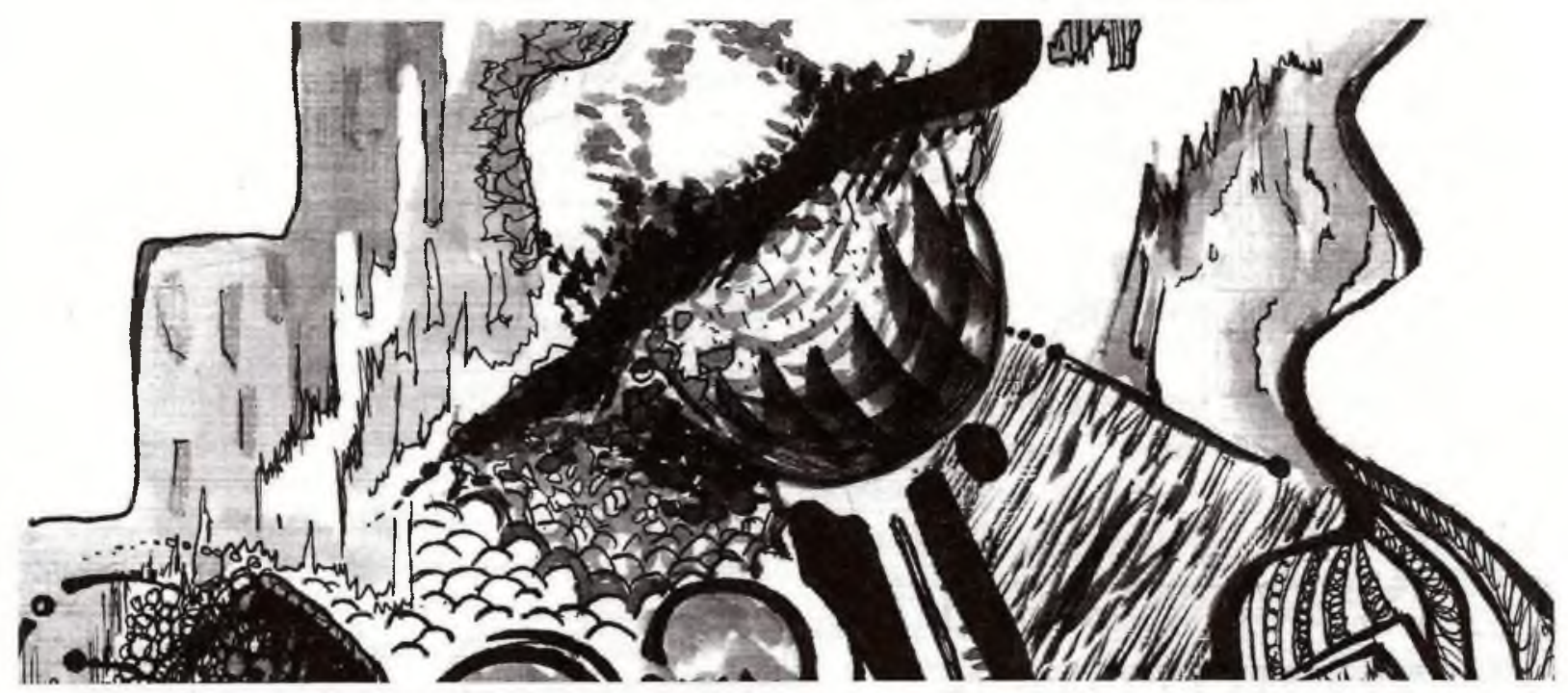

MG. OMAIRA ELIZABETH GONZÁLEZ
MG. DORIS CONSUELO PULIDO
DOCENTES UNIVERSIDAD DE LOS LLANOS

\section{RESUMEN}

El saber pedagógico es una noción que se incorpora en las propuestas curriculares y planes de estudio de los programas de licenciatura desde el decreto 272 de 1998, el cual establece los requisitos de creación y funcionamiento de los programas académicos en educación superior. De esta manera, en el país el currículo para todos los programas de licenciaturas se constituye desde los núcleos del saber pedagógico básicos y comunes: Realidades y tendencias sociales en la profesión docente; Historia y epistemología de la pedagogía; Educabilidad y Enseñabilidad.

La Escuela de Pedagogía de la Facultad de Ciencias Humanas en consenso entre los programas de licenciatura que oferta la universidad: diseñan el núcleo común pedagógico -los cuatro programas de licenciatura desarrollan los mismos cursos (unidades de contenidos, perfil de docente, competencias y evaluación) de tres de los núcleos que componen el saber pedagógico: Problemas históricos y epistemológicos de la pedagogía, Realidades y tendencias de la profesión docente y Educabilidad.

\section{PALABRAS CLAVE}

Núcleo común - Saber pedagógico - maestro pedagogía

\section{ABSTRACT}

Pedagogic knowledge is a notion imbedded in the curricular proposals and syllabi of the Licenciatura programs since the Decree 272 of 1998. This Decree sets out requirements needed to creation and functioning of academic programs in higher education. In this way, in our country the curriculum for all the licenciatura programs is constituted from the basic and common pedagogical knowledge nuclei: reality and social trends in the teaching profession; history and epistemology of pedagogy; teaching practice. 
The Pedagogy School of the Faculty of Human Sciences in agreement with the Licenciatura programs offered by the University, designs the pedagogical common nudeus. The four Licenciatura programs offer the same courses (content units, teacher profile, competencies and evaluations) of three of the nuclei that made up the pedagogical knowledge. Historic and epistemological problems of pedagogy, realities and tendencies of the teaching profession and teaching.

\section{KEY WORDS}

Common nucleus, pedagogical knowledge, professor, pedagogy.

\section{EXPERIENCIAS DEL NUCLEO COMÚN}

$\mathrm{E}$ I saber pedagógico es una noción que se incorpora en las propuestas curriculares y planes de estudio de los programas de licenciatura desde el decreto 272 de 1998, el cual establece los requisitos de creación y funcionamiento de los programas académicos en educación superior. De esta manera, en el país el currículo para todos los programas de licenciaturas se constituye desde los núcleos del saber pedagógico básicos y comunes: Realidades y tendencias sociales en la profesión docente; Historia y epistemología de la pedagogía; Educabilidad y Enseñabilidad.

El primero con preocupaciones en torno a los marcos globalizantes económicos, políticos, culturales, educativos, sociales y pedagógicos que plantean exigencias a las instituciones de formación de docentes más allá del dominio de unos saberes particulares; exhortándolas para que atiendan al reconocimiento y respeto de los $\mathrm{DDHH}$, al desarrollo de comportamientos de convivencia ciudadana y actitudes de conservación, cuidado y preservación de los bienes materiales y simbólicos culturales: regionales, nacionales y planetarios; así como a desarrollar competencias de pensamiento abierto, complejo y divergente frente al conocimiento, que le posibiliten actuar e interactuar aún en situaciones de incertidumbre; entre otros.

El núcleo de historia y epistemología de la pedagogía, en la intención de establecer un espacio curricular en los planes de las licenciaturas de reflexión en torno al saber fundante en la formación docente: la pedagogía como disciplina y como saber, desarrollando un dominio lingüístico, comunicativo, discursivo y conceptual de los conceptos, nociones, métodos y modelos con miradas hermenéuticas e históricas desde el interior de la disciplina, asumiendo crítica y reflexivamente aportes de otras áreas de conocimiento. Es desde la pedagogía que se establece el ethos del maestro, su identidad en la relación con el conocimiento y la cultura, son las instituciones de formación de docentes quienes establecen la impronta en el maestro como sujeto del saber pedagógico.

El núcleo de Enseñabilidad corresponde a la enseñanza de saberes específicos por área de formación disciplinar: matemáticas y física, educación física, pedagogía infantil, la cual sólo es posible en tanto el futuro docente conozca las condiciones de Enseñabilidad de la disciplina, parafraseando a Flórez Ochoa, el estatuto epistemológico de la misma; además debe acompañarse de una teoría o modelo pedagógico y establecer las condiciones particulares de los educandos en los procesos y niveles de pensamiento, estilos de aprendizaje, condiciones culturales, intereses y necesidades.

El núcleo de educabilidad se constituye en un lugar de construcción y desarrollo humano, tanto para sus docentes como estudiantes en formación, con base en principios de tolerancia, equidad, respeto a la diferencia, ternura e imprescindiblemente en la divergencia, la disidencia, la crítica reflexiva y los desacuerdos. En el que se valora lo humano, el amor por la vida singular y colectiva, lo iúdico, el placer, la razón, la voluntad y la cualidad por excelencia de la persona humana: la libertad. "Conocer lo humano es, principalmente, situarlo en el universo y a la vez separarlo de él. Cualquier conocimiento debe contextualizar su objeto para ser pertinente. "QQuienes somos?" es indispensable de un "¿Dónde estamos?" "¿de donde venimos?" "¿A dónde vamos?"70. 
Desde esta mirada los núcleos del saber pedagógico que corresponden a la invitación de la UNESCO desde el documento de Jacques Delors de una formación integral atendiendo a los cuatro pilares de la educación: aprender a ser, aprender a conocer, aprender a vivir juntos y aprender a hacer; fortaleciendo así lo común y lo esencial de la formación de maestros.

La Universidad de los Llanos en El Acuerdo 007 del 2002, por el cual se establece la Organización y Estructura Curricular, contextualiza las directrices del Decreto 272. La Escuela de Pedagogía de la Facultad de Ciencias Humanas en consenso entre los programas de licenciatura que oferta la universidad: Producción Agropecuaria, Matemáticas y física, Educación Física y Deportes y Pedagogía Infantil; diseñan el núcleo común pedagógico -los cuatro programas de licenciatura desarrollan los mismos cursos (unidades de contenidos, perfil de docente, competencias y evaluación) de tres de los núcleos que componen el saber pedagógico: Problemas históricos y epistemológicos de la pedagogía, Realidades y tendencias de la profesión docente y Educabilidad.

Estos cursos del núcleo común pedagógico (unidades de enseñanza-aprendizaje) se estructuran en la comprensión $y$ el compromiso de que la calidad de la educación está determinada principalmente, por la formación de los maestros y su práctica pedagógica. "La formación docente cubre diferentes ámbitos como la ubicación y comprensión del momento histórico en que se vive, los principios que rigen la educación, entre ellos el fortalecimiento de una democracia participativa, la preocupación por los valores, el desarrollo de la ciudadanía, la actitud de cambio, una cultura de diálogo y respeto por la diferencia, destacando en este contexto la dimensión ética, elemento esencial en la construcción de esa cultura y de un país cuya base fundamental, dadas las condiciones actuales, es la reconstrucción del tejido social, de la convivencia social y de la identidad"71. El propósito es consolidar una Escuela de pensamiento, una impronta de maestro, una comunidad de docentes que en la región constituya la conciencia pedagógica de la Orinoquia. De esta manera, el maestro ha de ser un Intelectual de la pedagogía que de cuenta de su saber y del saber pedagógico. La reflexión permanente sobre su quehacer permite la transformación de su práctica, se transforma él y transforma a los alumnos. Responsabilidad que le da el poder elaborar y producir un saber, el saber pedagógico.

La flexibilidad curricular, académica y administrativa de éste núcleo común fortalece la mirada interdisciplinaria hacia la pedagogía en las relaciones con la Enseñabilidad de las ciencias, la enseñanza y las didácticas particulares; así como la reconceptualización de la teoría pedagógica en los espacios sociales de práctica. "la flexibilidad se ha convertido en un aspecto clave de clima cultural y una metáfora clave para configurar una variedad de discursos mutuamente dependientes ${ }^{\prime \prime 2}$

Dinamiza diversos desarrollos en la comunidad docente de la Escuela: la conformación de grupos de estudio interdisciplinarios que abocan lecturas críticas fundamentales y básicas de documentos, autores y discursos propios de las problemáticas, nociones de la pedagogía y los saberes disciplinares; con progresivas elaboraciones de proyectos de investigación institucionales en el interés de establecer marcos historiográficos y cronológicos de los procesos educativos y pedagógicos para el municipio y el departamento. El fortalecimiento nocional, conceptual, metodológico y de problematización hacia los saberes específicos en articulación con la pedagogía y la didáctica, que permite colectivamente y como sujeto individual abordar los conflictos cognitivos en un proceso de deconstrucción, elaboración y reelaboración de los saberes. 
Discursos, registros y códigos de los núcleos del saber pedagógico que progresivamente circulan desde los diversos espacios formales institucionales: encuentros en clase, bibliotecas, centros de investigación, hacia los informales: diálogos, estudiantes-estudiantes, profesoresprofesores, estudiantes-profesores con manifestaciones en la cultura universitaria de procesos de recontextualización desde los marcos conceptuales y referenciales de las áreas de conocimiento a manifestaciones valorativas, comportamentales y actitudinales del ser $y$ hacer del docente en formación. Como lo afirma Olga Zuluaga, "el maestro como sujeto de saber y practica pedagógica fortalece los diálogos de la ciencia que enseña".

Espacios dialógicos conceptuales y afectivos de los sujetos del saber pedagógico que han posibilitado el acercamiento de las islas de la enseñanza de las disciplinas y la disminución en el engreimiento y el estatus superior en que se consideraban algunas de ellas; para conceptuar los núcleos de este saber pertenecientes al ser y deber ser en la formación integral de todos y cada uno de los estudiantes de los programas de licenciatura de la Unillanos.

Así mismo propicia desarrollos individuales y colectivos en los estudiantes y egresados en tanto como Escuela de Pedagogía se concibe el maestro como miembro activo de la comunidad de sujetos de saber pedagógico, traspasando las barreras de las particularidades de la enseñanza de los saberes específicos, los niveles escolares de formación y los objetos, conceptos y métodos de las disciplinas. Contextos socializantes y discursivos interdisciplinarios en la formación de un estudiante cuya práctica pedagógica responde a un área escolar específica (matemáticas y física, producción agropecuaria) posibilitando a mediano plazo los tránsitos para la constitución de comunidades pedagógicas de docentes en formación.
Rousseau, en su libro el Emilio plantea: "Haced a vuestro alumno atento a los fenómenos de la naturaleza, bien pronto lo haréis curioso; para alimentar su curiosidad, no os apresuréis jamás a a satisfacerla. Poned a su alcance las cuestiones y dejádselas resolver; que no sepa nada porque se lo habéis dicho, sino porque no lo comprendió el mismo: que no aprenda la ciencia, que la invente." Por tal razón Se establece en estos diálogos con pares estudiantes de diversas áreas de formación la comprensión continua que se amplia a una concientización, de las exigencias internas desde la disciplina y su enseñanza y externa desde otras disciplinas y enseñanzas así como de los contextos globalizantes comunicativos, tecnológicos, políticos, culturales hacia la función del maestro y los maestros en la transformación de la sociedad local y global, rompiendo los roles profesionalizantes en la formación de docentes. El estudiante hace una mirada hacia el exterior de su disciplina para dilucidar la racionalidad: es en la sinergia, en los encuentros y rupturas de las enseñanzas y los aprendizajes de los diversos y variados saberes en los espacios del quehacer docente en donde se forma y a la vez formará integralmente a sus próximos educandos.

En la dimensión emocional en el lenguajear parafraseando a Maturana, el convencimiento y reconocimiento en el otro-a y los otros-otras de seres diferentes pero plenamente humanos; con potencialidades y posibilidades de desarrollo desde lo personal y social en todos los educandos; reconocerse en diferencias y similitudes, en la seguridad que es con el otro y los otros-otras como se construye cultura en una sociedad diversa e incluyente.

Atendiendo los propósitos de esta mesa de trabajo de saber pedagógico ${ }^{73}$ cerraremos arbitrariamente la recontextualización de los núcleos del saber pedagógico en los programas de licenciatura

73 Concepto formulado por el grupo de historia de las prácticas pedagógicas en Colombia, quienes también establecen en el documento del CNA que se refiere, las reflexiones del núcleo de historia y epistemología de la pedagogía, y que corresponden significativamente tanto a los desarrollos de los proyectos de este grupo y a los diseños curriculares de los programas de licenciatura. Definen el saber pedagógico "conformado por los discursos a propósito de la enseñanza entendida en sentido práctico conceptual. Reúne los temas referentes a la educación, la instrucción, la pedagogía, la didáctica y la enseñanza. Es estrategia de análisis para historiar prácticas discursivas que han delimitado como sujeto de saber al maestro" en Zuluaga Olga Lucía. Pedagogía e historia. 1999 
de la Unillanos; a los desarrollos, desviaciones, preocupaciones, problematizaciones, consensos, acuerdos, discrepancias y rupturas del núcleo de historia y epistemología de la pedagogía.

Este núcleo junto con el de educabilidad y realidades y tendencias de la formación docente conforman el núcleo común pedagógico de la Escuela de Pedagogía -los cuatro programas de licenciatura desarrollan los mismos cursos-, lo componen en orden ascendente desde el primer semestre: Historia de la Pedagogía, Epistemología de la Pedagogía, Corrientes Pedagógicas y Contemporáneas en Colombia, Pedagogía, didáctica y conocimiento, Didáctica y Mediaciones Pedagógicas, Administración, currículo y legislación, Evaluación y proyecto de grado, Escuela siglo XXI y proyecto de grado; cada uno acompañado de la práctica investigativa, siendo prerrequisito la aprobación para el avance al siguiente curso.

Cursos que atendiendo a las reflexiones degrupos de docentes en torno a los núcleos del saber pedagógico ${ }^{74}$ han establecido contenidos del desarrollohistóricodelosconceptosarticuladores de la pedagogía ${ }^{75}$-enseñanza, formación, educación, aprendizaje; entre otros, se apoyan en lecturas de los griegos como pioneros de la reflexión y método en la educación, biografías y textos básicos y fundamentales de pedagogos como Vives, Comenio, Herbart, Pestalozzi y Rousseau quienes problematizaron el método, el objeto y los sujetos de saber en la pedagogía, estableciendo horizontes conceptuales en el desarrollo de esta disciplina según consideran algunos autores.

Se abordan temáticas que indagan acerca de los modelos y teorías pedagógicas, la didáctica general y particulares de los saberes disciplinares, la evaluación educativa, el análisis de Proyectos Educativos Institucionales y las mediaciones en los procesos pedagógicos. Las prácticas formativas en los espacios escolares desarrollan procesos de observación en la enseñanza, sistematización de experiencias, proyectos de aula, confrontación y análisis desde los marcos teórico-referenciales. Constituyen algunas estrategias pedagógicas el seminario alemán, las reseñas de textos, el seminario de autores, actas de las discusiones de clase, elaboración de ensayos, lectura y análisis de historias de vida de maestros y pedagogos.

Los programas de licenciatura particularmente el núcleo de historia y epistemología de la pedagogía forma al sujeto específico del saber pedagógico: el maestro, quien tiene como saber fundante en su formación la pedagogía como disciplina y como saber $^{76}$, disciplina en tanto se ha determinado su método, objetivo, discurso, campos de práctica, principios y propósitos. El estudiante en formación docente requiere apropiar estos elementos para comprender los lineamientos conceptuales históricos y epistemológicos que orientan y problematizan el presente de la pedagogía, a la vez que determinan el devenir de la misma en respuesta a las demandas de los entornos escolares, sociales y culturales.

Núcleo que debe establecer relaciones y distanciamientos con disciplinas y ciencias que también abordan con sus propios métodos, objetivos y estrategias las relaciones con el sujeto psicológico y social que aprende, la educación, el espacio escolar y la cultura. Saber pedagógico en tanto espacio flexible de conocimiento que contextualiza y recontextualiza los discursos, nociones, conceptos, prácticas de los sujetos que enseñan y condiciones particulares de los sujetos niños, jóvenes y adultos que aprenden.

Son los programas de formación de docentes en la implementación de los diseños curriculares en espacios dialógicos, constructivos, reflexivos y críticos los que determinan las delimitaciones del saber pedagógico: prácticas, discursos e instituciones. El sujeto del saber pedagógico en los espacios sociales y culturales establece las prácticas y discursos que conforman su saber, haciendo explícitos en su quehacer: las

74 CNA. Pedagogía y Educación. Reflexiones sobre el Decreto 272 de 1998, para la Acreditación Previa de Programas en Educación. Compilación documentos. Bogotá 1999.

75 lbíd. Pág. 38

76 ZULUAGA Olga Lucía. Pedagogía e Historia. La historicidad de la pedagogía. La enseñanza, un objeto de saber. Editorial Universidad de Antioquia. 1999. Pág. 145. 
teorías/modelos pedagógicos, la elaboración conceptual, la deconstrucción y construcción de su saber, las intuiciones, tanteos e ideologías que determinan la comprensión del objeto de saber de la pedagogía: la enseñanza. En ésta el maestro debe mostrar dominios y sinergias en los enfoques teóricos, el saber pedagógico y las características de la Enseñabilidad de la disciplina.

Los programas de licenciatura de la Escuela de Pedagogía siete años después de la implementación del núcleo de histórica y epistemología de la pedagogía en articulación con los otros núcleos del saber pedagógico encuentran desarrollos en los docentes que orientan los cursos de éste núcleo, son algunos de estos: la conformación de grupos de estudio interdisciplinarios -docentes y estudiantes- que establecen diálogos desde las regiones conceptuales de la pedagogía; apropiación de discursos, códigos, registros de la pedagogía como disciplina y saber y su uso en espacios académicos y socializantes; reflexiones, contextualizaciones de los marcos referencial y teórico-conceptual que le brindan un nuevo prisma a las prácticas formativas y de profesionalización; aproximaciones $y$ abstracciones de los conceptos fundamentales de la pedagogía en una metodología historiográfica; lectura de textos y formulaciones conceptuales de pedagogos que trazaron caminos en el desarrollo de la pedagogía y atisbo de miradas pedagógicas e interdisciplinares hacia las áreas de formación del saber específico. Los cursos regularmente están orientados por dos profesores uno del área disciplinar y otro del saber pedagógico que articulan las acciones y estrategias educativas en los espacios de práctica formativa.

También se encuentran rupturas conceptuales en los maestros: desconocen los métodos de estudio de los objetos de conocimiento propios de la pedagogía y asumen métodos, objetos y discursos provenientes de otras disciplinas sin recontextualización en la enseñanza; la historia de los pedagogos, su biografía, las ideas pedagógicas y su producción intelectual se convierten en un fin en sí mismas, repitiendo sus elaboraciones sin entenderlos en las condiciones históricas, políticas, sociales y culturales actuales, convirtiéndose en evaluación las fechas y nombres de sus textos; aún no se inician los procesos reflexivos en torno a las problematizaciones, tensiones y encuentros en las relaciones pedagogía y educación, pedagogía y enseñanza, pedagogía y sociedad y se expresa confusión en los discursos de los enfoques, modelos y teorías pedagógicas, se desconocen los principios pedagógicos ${ }^{77}$ que guían las prácticas formativas y profesionales así como los obstáculos epistemológicos ${ }^{78}$ de la pedagogía.

Por otra parte el concepto macro de enseñanza continúa encontrándose y resolviéndose desde la psicología o desde la intuición y no desde la pedagogía, lo que constituye una enorme debilidad en el dominio discursivo del saber pedagógico en los docentes y por supuesto en los estudiantes.

Núcleo/centro articulador de conocimientos, prácticas, emociones, creencias e ideología para la formación integral del futuro docente en las dimensiones del ser docente, el saber pedagógico y disciplinar, el saber hacer como sujeto del saber pedagógico en la enseñanza y el conocer del contexto social, cultural y político en su función social, en tanto que "se pregunta este núcleo por el objeto de la pedagogía, esto es por la constitución misma del campo de la educación y de la pedagogía y por las elaboraciones históricas sobre el discurso pedagógico en el cual se expresan la práctica de la instrucción y la práctica de la formación, así como sobre las instituciones formadoras de docentes ${ }^{\prime \prime 79}$

En el saber, apropiación de un discurso propio de la pedagogía, principalmente de los conceptos

\footnotetext{
77 Flórez Ochoa Rafael. Hacia una pedagogía del conocimiento. Editorial McGraw Hill. 1999. Pág. 115 -118 78 Ibíd. Introducción. Pág. XXVI

79 CNA. Pedagogía y Educación. Reflexiones sobre el Decreto 272 de 1998, para la Acreditación Previa de Programas en Educación. Compilación documentos. Bogotá 1999. Pág. 12
} 
articuladores en el espacio de socialización vital para el desarrollo del ser docente como es el grupo de pares, la comunidad académica institucional y la escuela; en el saber hacer desarrollo de propuestas pedagógicas en la institución educativa a través de proyectos de aula y apoyo de proyectos institucionales, adquisición de competencias pedagógicas y didácticas para hacer enseñable la disciplina a estudiantes particulares de la cultura.

En el ser por la construcción individual y social de maestro, en el reconocimiento del otro y de los otros diferentes pero plenamente humanos, en el sentido de una educación para todos y todas, en la creencia de la búsqueda de desarrollo personal y social de todos los educandos, en la conciencia de que en la educación se encuentra la posibilidad de apropiar el mundo subjetivo $y$ objetivo, la tecnología y la ciencia, en el derecho que le cabe a todos de transformar su realidad y mejorar su calidad de vida; en el convivir juntos en un entorno educativo y social en diferencias y similitudes, en la seguridad que es con el otro $y$ los otros como se construye cultura y sociedad.

El núcleo de historia y epistemología de la pedagogía que fortalece el estatuto de la función social del docente, y recibido con entusiasmo por la comunidades pedagógicas, e implementándose en las particularidades y autonomías de las facultades de educación y programas de formación docente y que estableció en los diseños curriculares espacios concretos de reflexión, discusión, lectura y análisis de los objetos de estudio y relaciones de la pedagogía; sin embargo encuentra en diferentes niveles de discursos y prácticas algunos tropiezos: los profesores de los programas se formaron en las facultades de educación las cuales privilegiaron los saberes específicos (educación física, preescolar, matemáticas y física) con elementos atomizados, desarticulados y descontextualizados del saber pedagógico, lo que plantea problematizaciones al docente quien debe enseñar este saber pedagógico ¿̨En dónde están formados los pedagogos? ¿Qué perfiles tienen? ¿Cómo orientar y valorar estos saberes en los estudiantes si ellos mismos carecen de estos referentes? ¿Cómo enseñar un saber sin los espacios discursivos sociales y disciplinares que hubieran posibilitado la deconstrucción y construcción individual y colectiva del conocimiento? ¿Qué debe hacer, conocer, ser el docente para responder a las demandas de ser un sujeto del saber pedagógico?

También las características mismas de la pedagogíaconsideradapordiversascomunidades de pedagogos y docentes como disciplina, ciencia, campo, disciplina en construcción, región, saber; genera confusión en sus discursos, prácticas, principios y métodos.

Por otra parte los estudiantes en las prácticas formativas y profesionales docentes, muestran una escisión entre los saberes de su formación: disciplinares, pedagógicos y de enseñanza y el quehacer de este saber pedagógico en la relación con los sujetos del conocimiento; en tanto reconocen estos marcos conceptualesreferenciales pero sin reelaborarlos en las prácticas pedagógicas, en donde aplican los modelos de enseñanza recibidos en su condición de estudiante (enseña como le enseñaron) sin las elaboraciones nocionales y conceptuales producto de su formación como docente.

Así mismo, un porcentaje significativo de estudiantes en la elaboración de propuestas de proyectos de grado o documentos de práctica profesional docente, limitan éstos a las problematizaciones desde los saberes particulares disciplinares, desconociendo los otros elementos lingüísticos y de relaciones entre las disciplinas y las prácticas vividas en los núcleos del saber pedagógico y particularmente la pedagogía como su saber fundante; situación que impele a preguntarnos ¿Por qué el estudiante hace esta ruptura? ¿Tienen los cuatro núcleos del saber pedagógico la misma importancia en los diseños curriculares? ¿Es la enseñabilidad el núcleo con mayor fortaleza en tanto los docentes tienen mayor dominio de la disciplina?

Por otra parte en las instituciones formadoras de docentes se empiezan a escuchar con fuerza e insistencia voces que reclaman aumentar los tiempos para los saberesespecíficosconargumentos de mayores desarrollos disciplinares los cuales son exigidos por el entorno social como apropiación del saber y para los estudios en postgrados de estos mismos estudiantes en formación. 
Presiones externas del estado en contravía con decisiones de otras instancias en el desconocimiento del estatuto del maestro $y$ de la pedagogía, cuando abre la posibilidad para profesionales de áreas diferentes a las licenciaturas de fungir como docentes, en tanto que con unas horas de capacitación se considera ya se han arropado con los saberes de la enseñanza. Se puede añadir a estos desaciertos las evaluaciones ECAES para los estudiantes de licenciatura en las cuales no es clara la valoración en lo pedagógico. Cabe señalar que los programas de licenciatura se encuentran entre los de menor solicitud de inscripción y matrícula en el país, correspondiendo a lo mostrado en la universidad de los Llanos.

Lo presentado entonces, muestra principalmente dos situaciones: lineamientos para los diseños curriculares de los programas de licenciatura que agrupan los saberes esperados en la formación integral del maestro y las barreras que limitan esta formación en los futuros docentes, estas con mayor importancia las que corresponden a los maestros. Situación que demanda acciones lideradas desde las instituciones formadoras de docentes, para constituir comunidades pedagógicas redes de docentes con los propósitos de problematizar, discutir, conceptualizar y escribir desde y sobre la pedagogía y el saber pedagógico. De otra manera, el descuidar este compromiso y retomando la mirada que hace la UNESCO a la educación superior, es necesario preguntarnos ¿Las facultades de educación, una especie en peligro de extinción?

\section{BIBLIOGRAFÍA}

CNA. Pedagogía y educación. Reflexiones sobre el decreto 272 de 1998, para la Acreditación Previa de Programas en Educación. Colección de documentos de Reflexión 2. Bogotá 1999

Delors Jacques. La educación encierra un tesoro. Informe a la UNESCO de la Comisión Internacional sobre la educación para el siglo XXI. Santillana Ediciones UNESCO. 1998

Flórez Ochoa Rafael. Hacia una pedagogía del conocimiento. Editorial MacGraw Hill. 1999
UNILLANOS. ProyectoEducativo Institucional. 2002

Zuluaga Olga Lucía. Pedagogía e historia. La historicidad de la pedagogía. La enseñanza, un objeto de saber. Editorial Universidad de Antioquia. 1999 Y otros. Pedagogía y epistemología. 